\title{
Distribution of Matrix Cracks in a Uniaxial Ceramic Composite
}

\author{
Chongdu Cho, ${ }^{\star}$ John W. Holmes, ${ }^{\star}$ and James R. Barber \\ Department of Mechanical Engineering and Applied Mechanics, University of Michigan, \\ Ann Arbor, Michigan 48109-2125
}

\begin{abstract}
Conventional shear-lag analyses of matrix cracking and debonding in uniaxial composites loaded in tension predict that the matrix stress varies only very slowly with position except near existing cracks. It therefore follows that the location of subsequent cracks is very sensitive to minor local variations in matrix strength, leading to significant statistical variation in crack spacing. This question is investigated using a discrete random process model of a composite and by direct experimental measurements of crack spacing. In the limit of a completely homogeneous composite, it is shown that the crack spacing distribution tends to an inverse square distribution between the theoretical maximum spacing and half that value. The random process model recovers this behavior in the limit and exhibits an approximately Weibull distribution of crack spacings when the matrix strength has significant variance. The theoretical predictions are compared with experimental results obtained for a unidirectional ceramic-matrix composite (SiC fibers in a calcium aluminosilicate matrix). The experimental results exhibit features similar to those predicted by the model and are compatible with a matrix strength whose standard deviation is of the order of $40 \%$ of the mean strength. An important point is that, with this magnitude of strength variation, the material exhibits a significant size effect and it is essential to take this into account in estimating the mean crack spacing from the corresponding mean matrix properties. [Key words: cracking, distillation, modeling, composites, stress.]
\end{abstract}

\section{Introduction}

$\mathbf{U}^{2}$ NIAXIAL composites under monotonic tensile loading have been the subject of numerous analytical and experimental studies. ${ }^{1-18}$ A typical loading scenario involves an initial linear, plane strain phase, followed respectively by matrix cracking, debonding between the fiber and the matrix near the matrix cracks, ${ }^{12-16}$ and ultimately fiber fracture.

As the load is increased, more matrix cracks are developed and hence the average crack spacing decreases. The process can be described by a simple shear lag model, which leads to an estimate of the maximum crack spacing as a function of load. ${ }^{2-4}$ However, the matrix stresses distant from the cracks at any given time are fairly uniform so the location of subsequent cracks and hence the crack spacing are subject to considerable statistical scatter. The stress level at which matrix cracking begins is influenced by variables such as residual stress state, fiber fraction, interfacial shear stress, and temperature. For a given composite or test specimen, slight differences in these variables (e.g., resulting from processing variations) can lead to a variance in matrix crack spacing even if the matrix strength is homogeneous.

A. G. Evans - contributing editor

Manuscript No. 197027. Received January 8, 1991; approved September 26,1991 .

*Member, American Ceramic Socicty.
The present paper is concerned with the question of predicting and measuring the statistics of matrix crack spacing in a uniaxial composite as a function of applied tensile load.

\section{The Model}

The composite microstructure is physically divided into rectangular cells (see Fig. 1) which consist of a single fiber or fiber bundle of diameter $d_{\mathrm{f}}$ embedded in matrix material of cross-sectional area $A_{\mathrm{m}}$. Notice that throughout the paper, the subscripts $f$ and $m$ will be used to denote fiber and matrix, respectively. The cross-sectional areas of fiber and matrix are proportional to the corresponding volume fractions $v_{\mathrm{f}}$ and $v_{\mathrm{m}}$, i.c.,

$$
\frac{A_{\mathrm{m}}}{A_{\mathrm{f}}}=\frac{v_{\mathrm{m}}}{v_{\mathrm{f}}}=\frac{1-v_{\mathrm{f}}}{v_{\mathrm{f}}}
$$

It follows that the total cross-sectional area of the cell is

$$
A=A_{\mathrm{m}}+A_{\mathrm{f}}=\frac{A_{\mathrm{f}}}{v_{\mathrm{f}}}=\frac{\pi d_{\mathrm{f}}^{2}}{4 v_{\mathrm{f}}}
$$

and if the mean tensile stress applied to the composite is $\sigma$, the tensile force transmitted by the cell is

$$
F=\sigma A=\frac{\sigma \pi d_{\mathrm{f}}^{2}}{4 v_{\mathrm{f}}}
$$

We consider the case where the load $F$ is applied to the fiber at the end of the cell, as shown in Fig. 2. If the cell is sufficiently long, plane strain conditions will develop in a central region, within which the applied tensile stress is divided between the fiber and the matrix in accordance with the volume fraction and the tensile moduli and there is no interfacial shear stress. In this region, fiber and matrix stresses will be given by

$$
\sigma_{\mathrm{f}}=\frac{E_{\mathrm{f}} \sigma}{E} ; \quad \sigma_{\mathrm{m}}=\frac{E_{\mathrm{m}} \sigma}{E}
$$

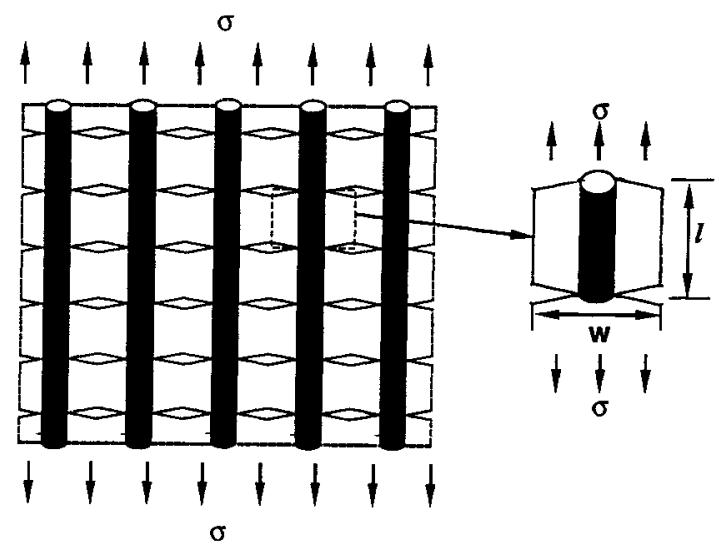

Fig. 1. Definition of the cell. 


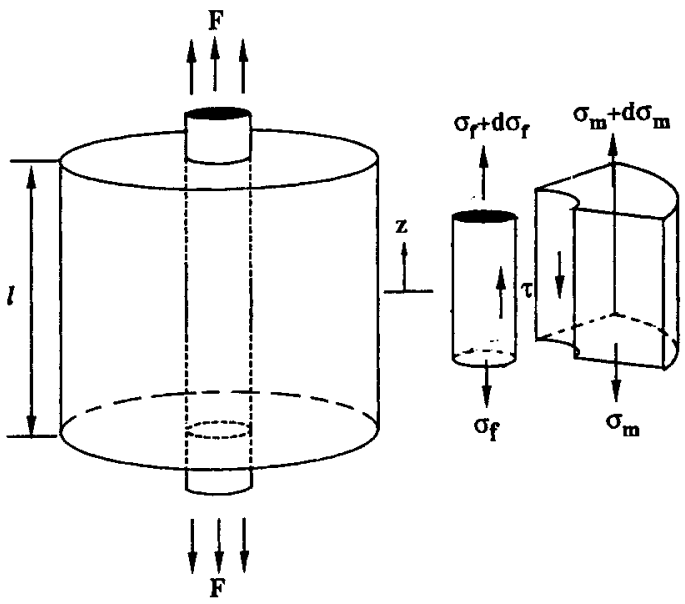

Fig. 2. Shear lag model used to estimate the shear stress distribution along the fiber-matrix interface.

where $E$ is the composite modulus defined by

$$
E=v_{\mathrm{f}} E_{\mathrm{f}}+v_{\mathrm{m}} E_{\mathrm{m}}
$$

\section{(1) Interfacial Shear Stress}

Near the ends, there will be a transition region in which load is transmitted from the fiber to the matrix by shear along the interface. The interfacial shear stress $\tau$ can be estimated using a "shear lag" theory, such as that of Aveston and Kelly. ${ }^{4}$ Using this approach, the real three-dimensional matrix and fiber stresses are replaced by their mean values and the elasticity of the interface is estimated on the basis of uniform shear loading of the matrix. This analysis (see Appendix A) predicts that $\tau$ decays exponentially with distance $z^{\prime}$ from the ends, i.e.,

$$
\tau=\frac{\lambda d_{\mathrm{f}} C \sigma}{4} \exp \left(-\lambda z^{\prime}\right)
$$

where the decay rate $\lambda$ is given by

$$
\lambda^{2}=\frac{8 G_{\mathrm{m}}}{C E_{\mathrm{f}} d_{\mathrm{f}}^{2}\left(\sqrt{v_{\mathrm{f}}}-v_{\mathrm{f}}\right)}
$$

$G_{\mathrm{m}}$ is the shear modulus of the matrix material and

$$
C=\frac{v_{\mathrm{m}} E_{\mathrm{m}}}{v_{\mathrm{f}} E}
$$

is a dimensionless constant.

The approximation inherent in the shear lag theory can be assessed by comparing Eq. (6) with the results of an axisymmetric finite element model (FEM) of the system (see Fig. 3). The predicted interfacial shear stresses agree with the FEM results within $5 \%$.

Equation (6) is appropriate if the length $l$ of the cell is sufficiently long for the transition regions at the two ends not to overlap, i.e., if $\lambda l \gg 1$. If this condition is not satisfied, a solution is readily obtained taking account of the interaction of the two transition regions. In this case, it is more convenient to utilize the symmetry of the cell by moving the origin to the center and defining $z=\frac{1}{2} l-z^{\prime}$. The appropriate expression for $\tau$ is then found as

$$
\tau(z)=-\frac{\lambda d_{\mathrm{f}} C \sigma}{4} \frac{\sinh (\lambda z)}{\cosh \left(\frac{1}{2} \lambda l\right)}
$$

while the corresponding mean matrix stress is

$$
\sigma_{\mathrm{m}}(z)=\frac{E_{\mathrm{m}} \sigma}{E}\left(1-\frac{\cosh (\lambda z)}{\cosh \left(\frac{1}{2} \lambda l\right)}\right)
$$

These expressions are shown in Fig. 4. In particular, wc note that the maximum shear stress occurs at the ends of the

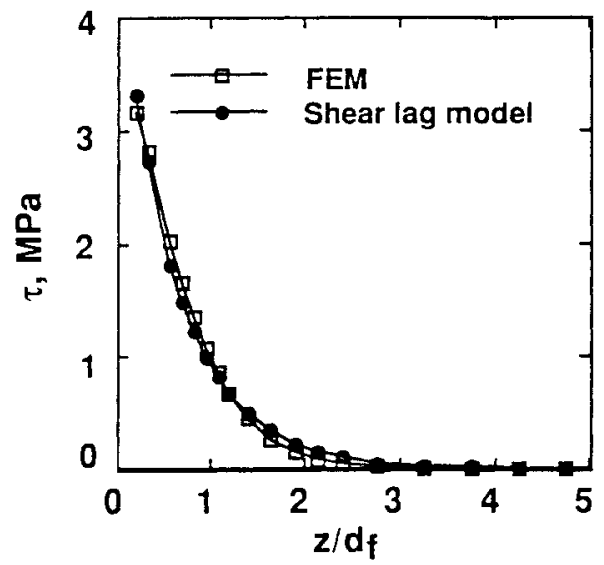

Fig. 3. Comparison between the interface shear stress calculated from the model, and finite element results $\left(E_{\mathrm{m}} / E_{\mathrm{f}}=0.3, \nu_{\mathrm{f}}=0.25\right.$, $\sigma_{\mathrm{t}}=25 \mathrm{MPa}$ at the crack plane).

unit cell $z= \pm \frac{1}{2} l$, while the maximum matrix stress occurs at the center, $z=0$. Thus, increasing the applied stress $\sigma$ will ultimately lead either to debonding between the fiber and the matrix near the ends or to the development of a matrix crack near the center of the cell.

\section{(2) Debonding}

In a recent analyses of debonding mechanisms in fiberreinforced ceramics, Hutchinson and co-workers ${ }^{5,13}$ assumed that debonding occurred when the mode II stress intensity along the fiber/matrix interface reached a critical value. However, in the present paper, since the emphasis is on the statistical distribution of matrix cracks, we adopt the simpler debonding criterion that debonding occurs when a critical value of interfacial shear strength, $\tau_{i}$, is reached. ${ }^{4}$ Once chemical debonding has occurred, relative slip between the fiber and the matrix is opposed by a constant frictional shear stress $\tau_{i}<\tau_{\text {i }}$.

Debonding will be initiated when the maximum stress defined by Eq. (9) reaches the value $\tau_{\text {i }}$, i.e., when

$$
\sigma=\sigma_{1} \equiv \frac{4 \tau_{\mathrm{i}}}{\lambda d_{\mathrm{f}} C} \operatorname{coth}\left(\frac{\lambda l}{2}\right)
$$

By symmetry, debonded regions of equal length $\delta$ will be developed at each end of the cell, so it is sufficient to consider the region $0<z<\frac{1}{2} l$. Furthermore, the interfacial shear stress in the bonded region satisfies the same differential equation and hence must be of the same form as Eq. (9).

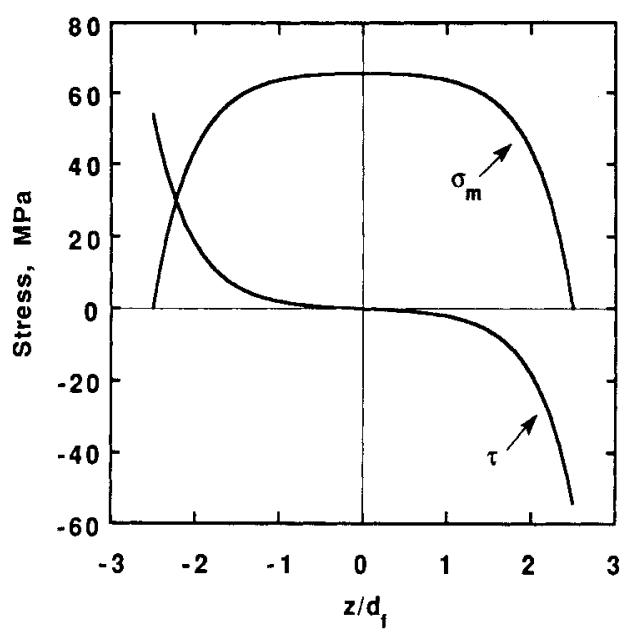

Fig. 4. Typical interfacial shear stress, $\tau$, and mean matrix stress, $\sigma_{\mathrm{m}}$, distributions 
Finally, if the loading is monotonic, the point $z=\frac{1}{2} l-\delta$ must be on the point of failing and hence $\tau\left(\frac{1}{2} l-\delta\right)=\tau_{\mathrm{i}}$. These conditions lead to the shear stress distribution

$$
\begin{aligned}
\tau & =-\tau_{\mathrm{f}} \operatorname{sgn}(z) \quad\left(\frac{l}{2}-\delta<|z| \leq \frac{l}{2}\right) \\
& =-\tau_{i} \frac{\sinh (\lambda z)}{\sinh \left[\lambda\left(\frac{1}{2} l-\delta\right)\right]} \quad\left(0 \leq|z| \leq \frac{l}{2}-\delta\right)
\end{aligned}
$$

Equilibrium conditions then define the relation between the length $\delta$ of the debonded zone and the applied stress $\sigma$ with the result

$$
\delta \tau_{f}+\frac{\tau_{\mathrm{i}}}{\lambda} \operatorname{coth}\left[\lambda\left(\frac{l}{2}-\delta\right)\right]=\frac{C \sigma d_{\mathrm{I}}}{4}
$$

It can be shown that $\delta$ increases monotonically with the applied stress $\sigma$, approaching complete debonding, $\delta=\frac{1}{2} l$, asymptotically as $\sigma \rightarrow \infty$.

If the argument of the hyperbolic cotangent is large compared with unity, Eq. (14) reduces to

$$
\delta \tau_{\mathrm{f}}=\frac{\operatorname{Co} d_{\mathrm{f}}}{4}-\frac{\tau_{\mathrm{i}}}{\lambda}
$$

agreeing with the debonding length equation of Aveston and Kelly. ${ }^{4}$

Once $\delta$ is determined from Eq. (14), the mean matrix stress can be found from equilibrium. In particular, we find

$$
\begin{aligned}
\sigma_{\mathrm{m}}(z)= & \frac{4 E_{\mathrm{m}}}{E C d_{\mathrm{f}}} \\
& \times\left\{\tau_{\mathrm{f}} \delta+\frac{\tau_{\mathrm{f}}}{\lambda}\left[\frac{\cosh \left[\lambda\left(\frac{1}{2} l-\delta\right)\right]-\cosh (\lambda z)}{\sinh \left[\lambda\left(\frac{1}{2} l-\delta\right)\right]}\right]\right\}
\end{aligned}
$$

in the central bonded region $|z| \leq \frac{1}{2} l-\delta$.

\section{(3) Matrix Cracking}

In the absence of debonding, i.e., for $\sigma \leq \sigma_{1}$, the matrix stress is given by Eq. (10), whereas for $\sigma>\sigma_{1}$, debonding occurs and the matrix stress in the central bonded region is given by Eq. (16).

In either case, matrix cracking will occur when $\sigma_{\mathrm{m}}$ at some point exceeds the tensile fracture strength $S_{\mathrm{m}}$ of the matrix matcrial. We assume that fracture will occur across the whole of the matrix phase, leading to the configuration of Fig. 1, in which there is now a central matrix crack bridged by the fiber. The matrix crack therefore essentially creates two new cells, each of which is loaded as in Fig. 2. Thus, by focusing on each of these cells in turn, using the symbol $l$ to denote the distance between adjacent matrix cracks, we can use the preceding analysis to describe the further development of the failure process.

(A) Crack Spacing without Debonding: If all regions of the matrix material have the same strength $S_{m}$, failure will occur at the midpoint $z=0$ of the unit cell, where $\sigma_{m}$ is a maximum. In the absence of debonding, the condition $\sigma_{\mathrm{m}} \leq S_{\mathrm{m}}$ and Eq. (10) yield the condition

$$
\frac{E_{\mathrm{m}} \sigma}{E}\left[1-\operatorname{sech}\left(\frac{1}{2} \lambda l\right)\right] \leq S_{\mathrm{m}}
$$

and hence

$$
l \leq \frac{2}{\lambda} \operatorname{sech}^{-1}\left(1-\frac{\sigma_{0}}{\sigma}\right) \equiv l_{\max }
$$

where

$$
\sigma_{0}=\frac{E S_{\mathrm{m}}}{E_{\mathrm{m}}}
$$

is the applied stress for which the plane strain matrix stress (4) would reach $S_{\mathrm{m}}$. The inequality (18) defines the maximum possible crack spacing $l_{\max }$ as a function of the applied stress $\sigma$ if there is no debonding.

(B) Crack Spacing with Debonding: When debonding occurs, the maximum matrix stress is given by Eq. (16) with $z=0$, i.e.,

$$
\sigma_{\mathrm{m}}(0)=\frac{4 E_{\mathrm{m}}}{E C d_{\mathrm{f}}}\left\{\tau_{\mathrm{f}} \delta+\frac{\tau_{\mathrm{i}}}{\lambda}\left[\frac{\cosh [\lambda(l / 2-\delta)]-1}{\sinh [\lambda(l / 2-\delta)]}\right]\right\}
$$

Two qualitatively different cases can be distinguished. If $\tau_{i} \leq 2 \tau_{\mathrm{f}}, \sigma_{\mathrm{m}}(0)$ increases monotonically with $\sigma$ as debonding progresses and the maximum crack spacing $l_{\max }$ is defined implicitly by the equation

$$
\begin{aligned}
\cosh \left[\lambda\left(l_{\max } / 2-\delta\right)\right] & -\frac{\lambda}{\tau_{\mathrm{i}}}\left(\frac{C \sigma_{\|} d_{\mathrm{f}}}{4}-\delta \tau_{\mathrm{f}}\right) \\
& \times \sinh \left[\lambda\left(l_{\max } / 2-\delta\right)\right]=1
\end{aligned}
$$

where $\delta$ is determined from Eq. (14) as a function of $\sigma$.

If $\tau_{i}>2 \tau_{f}$, Eq. (20) reaches a maximum before debonding is complete, at an applied stress level

$$
\begin{aligned}
\sigma= & \frac{4 \tau_{\mathrm{f}}}{C d_{\mathrm{i}}}\left[\frac{l}{2}+\frac{1}{\lambda} \cosh ^{-1}\left(\frac{\tau_{\mathrm{i}}}{\tau_{\mathrm{f}}}-1\right)\right. \\
& \left.+\frac{1}{\lambda}\left(\frac{\tau_{i}}{\tau_{\mathrm{f}}}-1\right) \sqrt{\frac{\tau_{\mathrm{i}}}{\tau_{\mathrm{i}}-2 \tau_{\mathrm{f}}}}\right] \equiv \sigma_{2}
\end{aligned}
$$

Debonding will then continue in the range $\sigma>\sigma_{2}$, but no further matrix cracking will occur, the final value of $l_{\max }$ being that obtained by substituting $\sigma_{2}$ for $\sigma$ in Eq. (21).

(C) Debonding After Matrix Cracking: Immediately after a matrix crack is formed, some debonding will occur immediately in regions adjacent to the new crack (i.c., the central crack in Fig. 4). However, it can be shown from Eq. (14) that $\delta$ decreases monotonically with decreasing $l$ at constant $\sigma$ and hence the new debonded regions will be smaller than those produced in the original uncracked cell. As the applied stress $\sigma$ is increased, debonding will occur only at the newly fractured ends of the cells until each subcell has equal debonded regions at each end, after which the process will be described by the preceding analysis.

Additional matrix cracking will not occur until debonding has progressed beyond the stage developed in the original cell. To demonstrate this, we note that Eq. (21) defines a relation between $\delta$ and $l$ at the stress level required for matrix cracking. Differentiating this expression with respect to $l$ shows that $\partial \delta / \partial l<0$ for all values of the parameters, provided that $\tau_{\mathrm{i}}<2 \tau_{\mathrm{r}}$. Thus, the extent of the debonded region increases as the cell length decreases. The same conclusion can be established for the case $\tau_{i}>2 \tau_{f}$, making use of the fact that no matrix cracking occurs for $\sigma>\sigma_{2}$

\section{Effect of Strength Statistics}

At any given load, the crack spacing will generally be less than the value $l_{\max }$ defined in the previous section. For example, the occurrence of the first crack immediately reduces the crack spacing to one half of its original value, at which it will remain until the stress level has increased sufficiently for the matrix stress in the new half-length cells to reach $S_{\mathrm{m}}$. With a matrix of completely homogeneous strength, this argument would define a series of critical stresses at which the number of cracks increased by a factor of 2 , while the crack spacing was halved.

However, we note from Fig. 4 and Eq. (10) that as long as $l \gg d_{\mathrm{f}}$, the matrix stress curve is very flat in the central region and hence comparatively minor statistical variations in matrix strength through the composite will be sufficient to destroy this symmetrical scenario. In general, we might an- 
ticipate a distribution of crack spacings (or instantaneous unit cell lengths) mostly in the range $l_{\max }>l>\frac{1}{2} l_{\max }$. Furthermore, as long as the crack spacing satisfies the condition $\lambda l \gg 1$, the matrix stress at the center of the cell is approximately equal to the plane strain value of Eq. (4). Thus, when the applied stress reaches $\sigma_{0}$ (see Eq. (20)), the uniform strength model predicts that sufficient matrix cracks would form instantaneously to reduce the cell length until $\lambda l$ is of the order unity. ${ }^{\dagger}$

To investigate these questions in more detail, we shall develop a numerical algorithm to describe the progressive damage of a composite specimen with a random distribution of matrix strength.

\section{(1) Discrete Simulation of the Specimen}

We suppose that the matrix material contains a random distribution of microdefects of various dimensions and orientations. The fracture strength $S(V)$ of any homogeneously loaded volume $V$ of matrix material will depend on the weakest defect contained within $V$ and hence the material strength will exhibit a size effect, since the probability of a defect of any given strength occurring within $V$ increases with $V$.

If we write $P(V, S)$ for the probability that a volume $V$ does not contain any defects weaker than $S$, it follows that the corresponding probability $P(n V, S)$ for a larger specimen of volume $n V$ must be

$$
P(n V, S)=[P(V, S)]^{n}
$$

since the larger specimen might be considered as a set of $n$ similarly loaded specimens of size $V$.

It is important that the statistical description of the matrix material used in the simulation should preserve this characteristic, since otherwise, changes in the scalc of the discretization would affect the results. ${ }^{\ddagger}$ The simplest distribution which preserves its form under the operation described by Eq. (23) is the two-parameter Weibull distribution, defined by

$$
P(V, S)=\exp \left[-\left(S / S_{V}\right)^{h}\right]
$$

where

$$
S_{V}=S_{0}\left(V_{0} / V\right)^{1 / b}
$$

and $S_{0}$ is defined for a reference volume $V_{0}$, such that $P\left(V_{0}, S_{0}\right)=0.368$.

Equations (24) and (25) show that the reference strength $S_{V}$ and hence the mean or median strength of the elements falls with $V$ as we should expect, but the shape parameter $b$, which is related to the dimensionless standard deviation of the distribution, is not affected by $V$.

We simulate a specimen with a random strength distribution by partitioning the initial length $l$ of the cell of Fig. 2 into 5000 equal elements and using a random number generator to assign a strength $S_{i}$ to each element $i$ in accordance with the above distribution function. The reference volume $V_{0}$ is taken to be that corresponding to a length $\lambda^{-1}$ of the specimen.

The equations of Section II are then used to describe the progressive damage of the specimen under monotonic loading. The matrix stress varies monotonically through each element of the cell except for the central element in the case of a cell with an odd number of elements. Failure will therefore generally be predicted at the interface between elements and will result in the division of one cell into two cells, not necessarily of equal lengths.

\footnotetext{
${ }^{\dagger}$ This explains why we must use the exact analysis (Refs. 9 and 10 ) rather than the approximate expression (6) to describe the process of matrix cracking, since Eq. (6) is only appropriate as long as $\lambda l \gg 1$.

*Even in this case, a discrete random process simulation requires that the element size be large compared with the median defect size. Otherwise, the strength of adjacent elements will be correlated and it would be nccessary to specify an autocorrelation function or spectral density for the material strength.
}

(A) Fully Bonded Algorithm: In the absence of debonding, the process can be analyzed without iteration, since, for any given crack configuration, the stress $\sigma_{i}$ at any node $i$ is a linear function of the applied load $F$. Denoting the stress due to a unit load as $\sigma_{i}^{*}$ we have $\sigma_{i}=F \sigma_{i}^{*}$. Failure will then occur first at the node which has the highest value of the ratio

$$
R_{i}=\frac{\sigma_{i}^{*}}{S_{i}}
$$

where $S_{i}$ is the strength of the weaker of the two elements which meet at $i$. Denoting this weakest node by $j$, the corresponding fracture load is then

$$
F=\frac{S_{j}}{\sigma_{j}^{*}}
$$

The occurrence of a new crack changes the crack configuration of the specimen and necessitates the calculation of a new set of values $\sigma_{i}^{*}$, after which the same procedure can be followed to determine the location and load for the next crack.

(B) Partial Debonding: When debonding occurs, the process is nonlinear and must be analyzed iteratively. This is conveniently done by increasing the applied load in small increments, using the solution of Section II to calculate the instantaneous matrix stress $\sigma_{i}$ at each node. Whenever the value of $\sigma_{i}$ reaches $S_{i}$ at any node, a crack is introduced at that node and the procedure is continued.

\section{(2) Theoretical Results}

We first consider the case where there is no debonding and the matrix strength has a Weibull distribution with shape factor $b=63.4$. With this value, the standard deviation $\hat{S}$ is only $2 \%$ of the corresponding mean strength $\bar{S}$ and hence the matrix is relatively homogeneous in strength.

Figure 5 shows the dimensionless mean crack spacing $\lambda \bar{l}$ and the dimensionless constitutive law as the applied tensile stress is increased. The reference stress $\sigma_{0}$ in this normalization is defined by Eq. (19) with $S_{\mathrm{m}}=S_{0}$. As predicted, multiple cracking occurs over a fairly small load range near $\sigma=\sigma_{0}$ until the dimensionless mean spacing is of the order unity. This causes a region of low incremental modulus in the constitutive law, as discussed by Cooper and Silwood. ${ }^{3}$

The dotted lines in Fig. 5 define the maximum crack spacing $l_{\max }$ and the minimum $l_{\text {max }} / 2$, as defined by Eqs. (18) and (19) with $S_{\mathrm{m}}=S_{0}$. The actual mean spacing lies within these limits and is reasonably well approximated by the expression $0.7 l_{\text {midx }}$.

As matrix cracking proceeds, the distribution of crack spacings changes character, as shown in Fig. 6. It is shown in Appendix B that the distribution which preserves its form with increasing load when the matrix strength is completely homogeneous corresponds to a distribution such that the cumulative probability of $l<x$ is $2-l_{\max } / x$, where $l_{\max }$ is

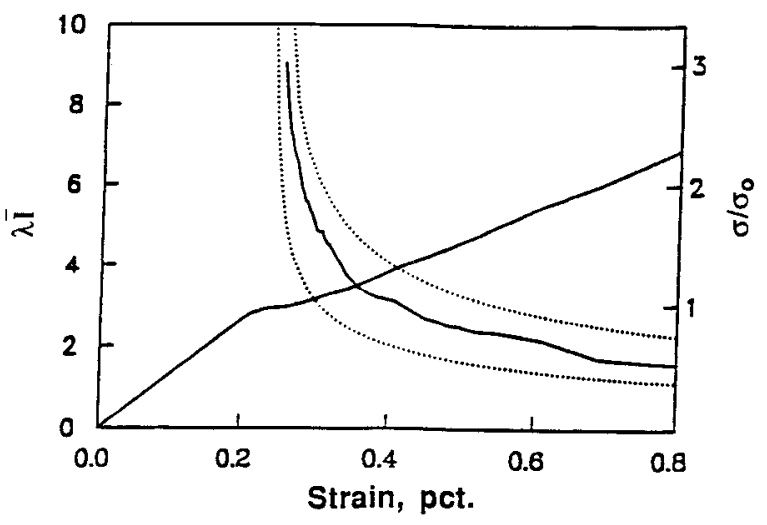

Fig. 5. Stress-strain relation and mean crack spacing predicted by the statistical model with $\hat{S}=0.02 \bar{S}$. 


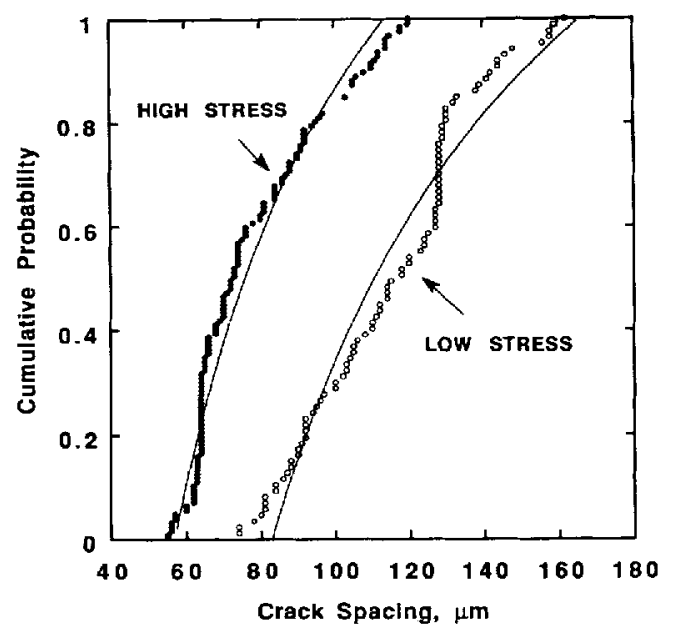

Fig. 6. Crack spacing vs cumulative probability for $0^{\circ}$-Nicalon/ CAS-II composites ( 40 vol\% fibers) for two different applied loads. The solid lines correspond to the crack spacing distributions predicted for a matrix of homogeneous strength. The points were obtained from the discrete simultation of Section III(1) using a standard deviation in matrix strength of $0.02 \bar{S}$.

given by Eq. (18). This is shown as a solid line in Fig. 6 and is clearly a good approximation to the discrete results for large stresses. This distribution also predicts that the mean crack spacing will be

$$
\bar{l}=l_{\max } \ln 2
$$

which is close to the mean value obtained from the simulation and shown in Fig. 5.

Throughout the load range, significant numbers of spacings arc obtained throughout the range $l_{\max }>l>l_{\max } / 2$, resulting in a standard deviation $\hat{l} \geq 0.2 \bar{l}$. Thus, the relatively flat matrix stress curve for $\lambda l \gg 1$ results in a large standard deviation for crack spacings, even when the matrix strength shows little statistical variation.

Similar results were obtained using Weibull matrix strength distributions with larger normalized standard deviations, corresponding to smaller values of $b$. The constitutive law and mean crack spacing are shown in Fig. 7 for $\hat{S}=0.4 \bar{S}$, corresponding to $b=2.725$. Notice that the mean crack spacing now falls below the limit defined by Eq. (18) at lower stress levels. This is essentially another manifestation of the size effect, which is very significant for materials with larger variance in strength. Incremental cracking in a large cell is dominated by occasional weak regions of matrix, rather than by the bulk of material near the mean strength. However, as the crack spacing is reduced, the probability of such a wcak region

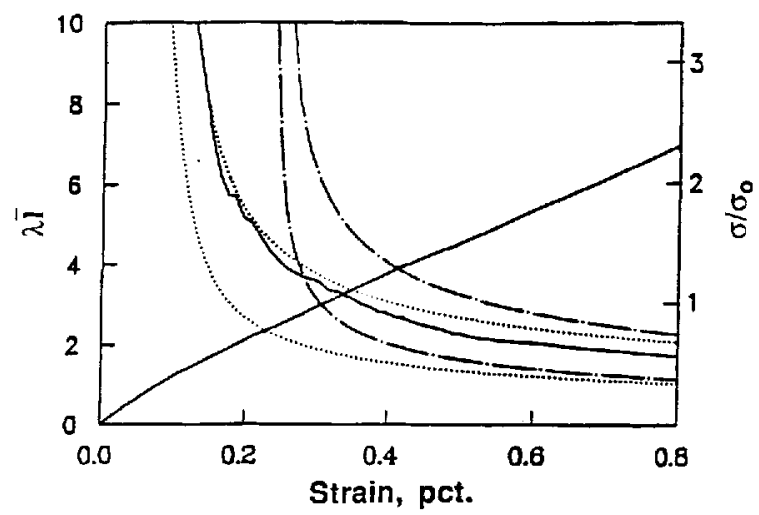

Fig. 7. Stress-strain relation and mean crack spacing predicted by the statistical model with $\hat{S}=0.4 \bar{S}$. Bounds defined by Eq. (18) are shown with $S_{m}=S_{0}(-\cdot-)$ and incorporating the size effect through Eq. (29) (․). occurring in any given cell is reduccd, causing the mean spacing to fall less rapidly than the theoretical value. The dependence of mean matrix strength on specimen size is taken into account in the definition of the Weibull probability (Eqs. (24) and (25)), through the occurrence of the specimen volume, $V$, in Eq. (25). We would therefore expect to obtain a better estimate of the mean crack spacing by equating $S_{\mathrm{m}}$ in Eq. (19) to the strength $S_{V}$ of a reference volume $V_{1}$ rclated to the current value of $l_{\text {max }}$. We then obtain

$$
S_{m}=S_{0}\left(V_{0} / V_{1}\right)^{1 / b}=S_{0} /\left(\lambda l_{\max }\right)^{1 / b}
$$

from Eq. (25), where we recall that the reference volume $V_{0}$ corresponds to a length $\lambda^{-1}$ of the specimen (see Section III(1)). Equations (18), (19), and (29) can be solved implicitly for $l_{\max }$ as a function of $\sigma$. The resulting limits $l_{\max }$, $l_{\text {max }} / 2$ are shown as dotted lines in Fig. 7 and clearly give better bounds on the crack spacing distribution.

For all the matrix strength distributions considered, the normalized standard deviation of crack spacing $\hat{l} \bar{l}$ decreases with applied stress, but tends to a limit at high stress as shown in Fig. 8. This limit is shown in Fig. 9 as a function of $b$ or $\hat{S} / \bar{S}$. As we might expect, an increase in the variance of matrix strength causes a corresponding increase in variance of crack spacing, but the effect is relatively modest. Notice that as $\hat{S} \rightarrow 0$, we recover the limiting result $\hat{l}=0.2 \bar{l}$ proved in Appendix B. When the matrix strength variance is significant--e.g., for $\hat{S}>0.2 \bar{S}-$ the distribution defined in Appendix B ceases to be a good approximation to the crack spacing distribution. However, in this range good correlations $(\approx 0.95)$ are obtained with a two-parameter Weibull plot.

The effect of debonding was assessed by repeating certain of these calculations using the equations of Section II(3)(B), with $\tau_{f}=0.5 \tau_{i}$. Results for $\hat{S}=0.4 \bar{S}$ are shown in Fig. 10 . The principal effects of debonding are to extend the region of low incremental modulus near $\sigma=\sigma_{\eta}$ and to reduce matrix cracking by reducing the ability of the interface to transmit load from the fibers to the matrix.

In fact, matrix cracking ceases and the mean crack spacing remains constant for $\sigma>1.1 \sigma_{i}$. The limiting value of crack spacing is sensitive to the values assumed for $\tau_{i}$ and $\tau_{f}$, increasing as cither of these quantities is reduced. As in the fully bonded case, the bounds defined using Eq. (29), which incorporates the size effect, are better than those based on the mean value $S_{0}$.

\section{Experimental Observations}

For comparison with the theorctically predicted trends, the instantaneous crack spacing in a 16-ply unidirectional

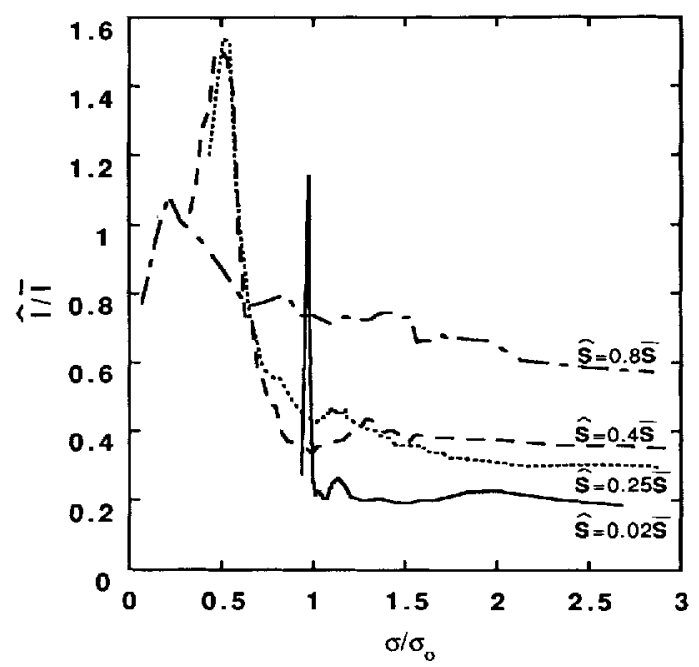

Fig. 8. $\hat{l} \bar{l}$ vs $\sigma / \sigma_{0}$ for matrix strength standard deviation $\hat{s}=$ $0.02 \bar{S}(-), 0.25 \bar{S}(\cdots), 0.4 \bar{S}(--)$, and $0.8 \bar{S}(-\cdots)$. 


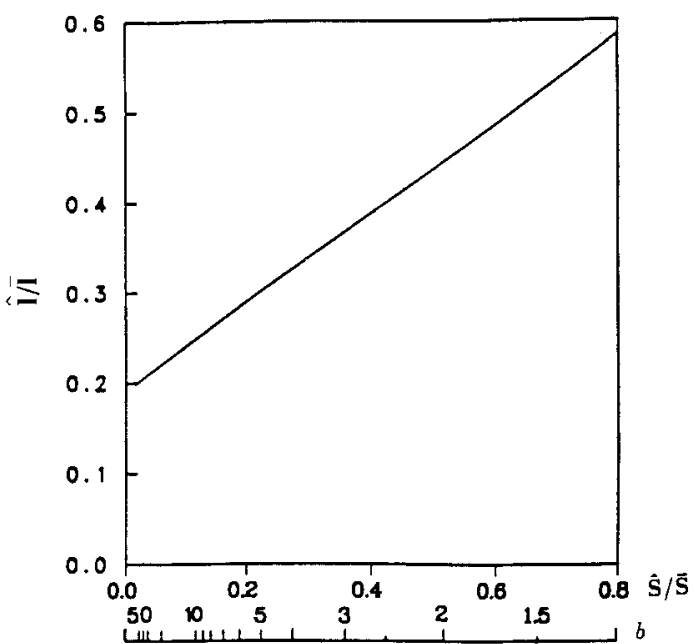

Fig. 9. Normalized standard deviations of crack spacing vs normalized standard deviations of matrix strength.

Nicalon/calcium aluminosilicate composite (hereafter referred to as $\mathrm{SiC}_{\mathrm{f}} / \mathrm{CAS}$ ) was determined as a function of applied tensile load. The Nicalon fibers, which have a nominal diameter of $8 \mu \mathrm{m}$, were processed in the form of 500 -tow fiber bundles. The composite was manufactured by hot-pressing. The nominal fiber fraction after hot-pressing was 40 vol\% (material obtained from Corning Glass Works, Corning, NY; the Corning Glass Works designation for this composite is $\left.[0]_{16} \mathrm{SiC}_{\mathrm{f}} / \mathrm{CAS}-\mathrm{II}\right)$.

\section{(1) Specimen Preparation and Mechanical Testing}

Edge-loaded tensile specimens (Fig. 11) were machined from $\mathrm{SiC}_{t} / \mathrm{CAS}$ billets using diamond tooling. Specimens were removed from the billets with the fiber axis parallel to the loading axis of the specimen. To allow acetate-film replicas of surface cracking to be obtained, the specimen edges were polished with diamond paste to a $0.5-\mu \mathrm{m}$ finish.

Tensile testing was conducted at room temperature on a 100-kN-capacity MTS Model 810 servohydraulic load frame. To minimize the introduction of bending strains in the test specimen, which would influence crack formation, the specimen, grips, and servohydraulic load frame were carefully aligned before testing. For the stress levels examined (130 to $360 \mathrm{MPa}$ ), the bending strain was less than $1.2 \%$ of the applied axial load (determined in accordance with ASTM Standard E1012-84 ${ }^{22}$ ).

The monotonic tensile behavior of the composite was determined under load control, at a constant rate of $100 \mathrm{MPa} / \mathrm{s}$. To determine crack spacing as a function of applied load, a specimen was sequentially loaded, from 130 to $360 \mathrm{MPa}$, in

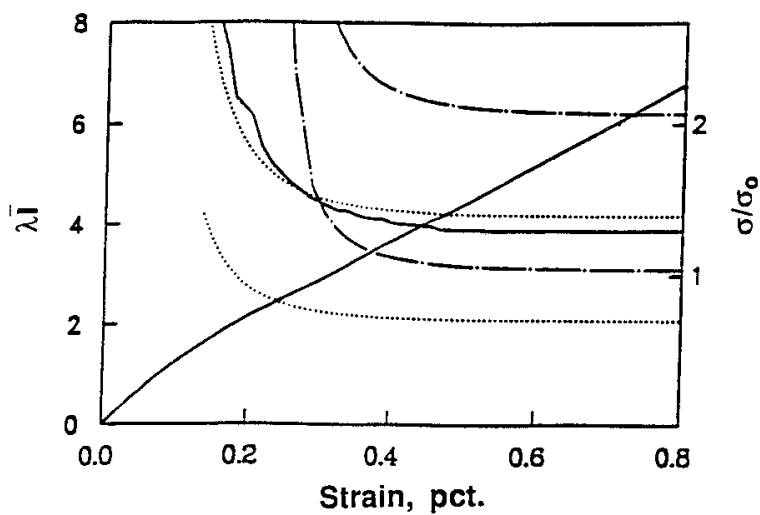

Fig. 10. Stress-strain relation and mean crack spacing predicted by the statistical model with $\hat{S}=0.4 \bar{S}, \tau_{\mathrm{f}}=0.5 \tau_{\mathrm{i}}$, and $\tau_{\mathrm{i}}=0.4 \sigma_{i j}$. Bounds defined by Eq. (18) are shown with $S_{\mathrm{m}}=S_{0}(-\cdot-)$ and incorporating the size effect through $\mathrm{Eq} .(29)(\cdots)$.

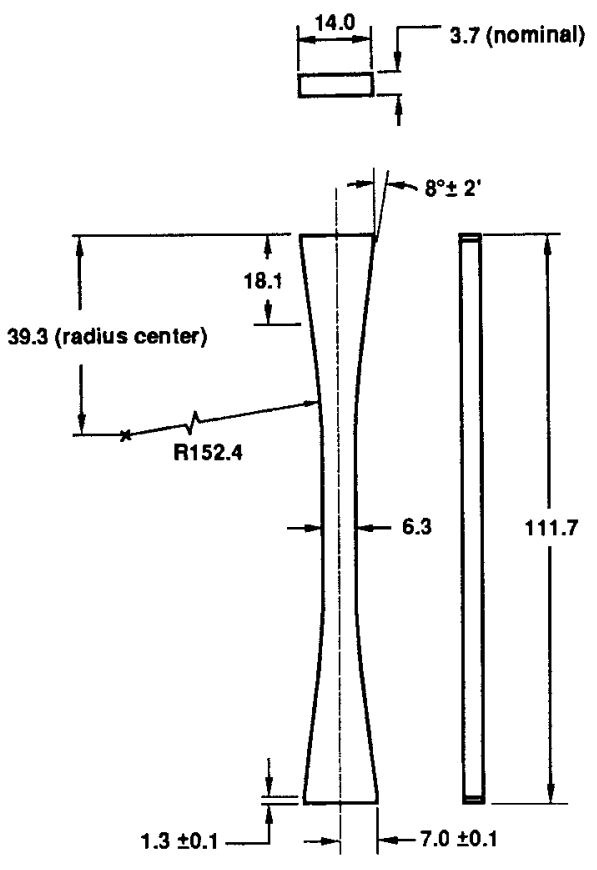

Unit : mm

Fig. 11. Edge-loaded tensile specimen ${ }^{19}$ used to determine crack spacing as a function of nominal applied stress. Crack spacing was determined by surface replicas taken along the specimen edge.

10-MPa steps (during loading to $360 \mathrm{MPa}$, the specimen failed). Preliminary tests indicated that both the crack density and maximum stress attainable during the loading and unloading experiments were strongly dependent on the particular loading sequence which was used. For example, loading to a desired stress level and holding at that stress while obtaining replicas resulted in a time-dependent increase in matrix cracks throughout the hold period. A similar time-dependent change in crack spacing under a static loading has been observed by Shuler et al. ${ }^{21}$ in experiments with $[0 / 90]_{4 S} \mathrm{C}_{\mathrm{f}} / \mathrm{SiC}$ composites. To minimize the influence of time-dependent inelastic deformation on crack spacing, the specimens were loaded and unloaded at a rate of $100 \mathrm{MPa} / \mathrm{s}$. At the end of each unloading ramp, the stress was maintained at $10 \mathrm{MPa}$ to provide further crack definition while taking the surface replicas.

\section{(2) Experimental Results}

The monotonic tensile behavior of the $\mathrm{SiC}_{\mathrm{f}} / \mathrm{CAS}$ composite is shown in Fig. 12. The composite exhibits linear behavior to approximately $250 \mathrm{MPa}$, followed by nonlinear behavior, with failure occurring at approximately $410 \mathrm{MPa}$.

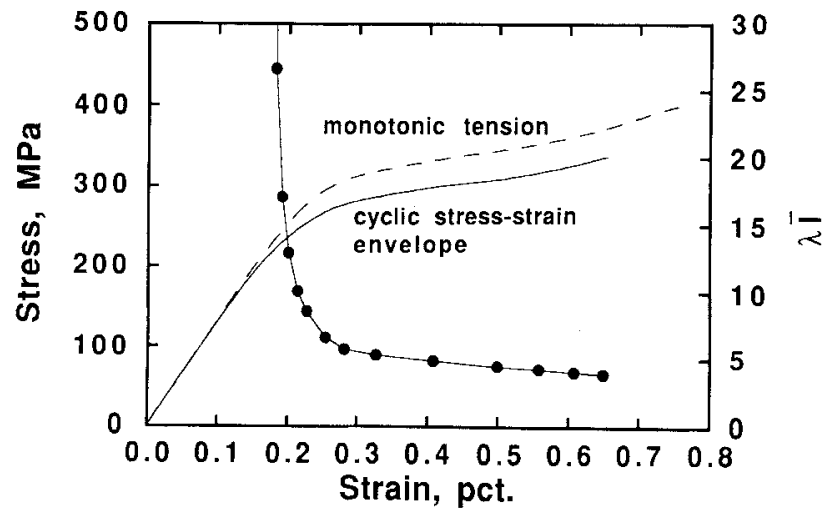

Fig. 12. Monotonic tensile behavior of $[0]_{16}-\mathrm{SiC}_{f} / \mathrm{CAS}$ composite at ambient temperature. Also shown is the stress-strain envelope obtained during the cyclic loading/unloading experiments, and the dimensionless mean crack spacings $\lambda \bar{l}$ which were experimentally determined $\left(\lambda=2.2 \times 10^{4} \mathrm{~m}^{-1}\right)$. 
Also shown in Fig. 12 is the envelope obtained by plotting the tips of the cyclic stress-strain curves obtained during the sequential loading/unloading cxperiments. Because of the presence of matrix cracks, the cyclic stress-strain envelope falls below the monotonic curve. Matrix cracking also significantly lowers the ultimate strain and stress at which failure occurs.

During the sequential loading/unloading experiments, random matrix cracks were first observed at a stress of $160 \mathrm{MPa}$, which is significantly below the stress of $260 \mathrm{MPa}$ at which a detectable change in specimen compliance is found during monotonic loading. Thus, from the cyclic experiments, $\sigma_{\theta}$ is expected to be approximately $160 \mathrm{MPa}$. The determination of a precise value for $\sigma_{0}$ would requirc conducting experiments on monolithic calcium aluminosilicate. Currently there are no consistent techniques which can be used to process monolithic calcium aluminosilicate with a composition similar to that used in $\mathrm{SiC}_{\mathrm{i}} / \mathrm{CAS}$ composites..$^{23}$

Figure 12 shows that the dimensionless mean crack spacing levels off at a value of around $\lambda \bar{l}=4$ at high values of applied stress. This limiling value can be used to deduce information about the interfacial shear stress, provided a plausible valuc can be assumed for the ratio $\left(\tau_{1} / \tau_{i}\right)$ between the frictional and the bonding shear strength. Taking $\sigma_{0}=160 \mathrm{MPa}$ ( $\mathrm{scc}$ above) and $\tau_{\mathrm{i}} / \tau_{\mathrm{i}}=1$, the model predicts a value $\lambda \bar{l}=4$ for $\tau_{i}=27 \mathrm{MPa}$. Alternatively, with $\tau_{\mathrm{f}} / \tau_{\mathrm{i}}=0.5$, the same prediction requires $\tau_{\mathrm{r}}=27 \mathrm{MPa}$. Thus, in this range, the predicted value of $\tau_{f}$ is not very sensitive to the assumed ratio. The predicted value for $\tau_{f}$ is in line with estimates from other sources. For example, from fiber pushout experiments Wang and Parvizi-Majidi ${ }^{24}$ estimated that the interfacial shear $\tau_{i}$ is primarily frictional in nature with the range of 13 to $18 \mathrm{MPa}$. A promising approach which is being utilized to determine frictional shear involves correlating the temperature rise which occurs during cyclic loading with the energy dissipation through frictional sliding of fractured fibers. ${ }^{25-28}$ This approach will provide a statistical mean for $\tau_{f}$. Preliminary estimates show an initial interfacial shear stress of approximately $15 \mathrm{MPa}$, which decreases to approximately $5 \mathrm{MPa}$ after long duration cyclic loading. ${ }^{2 x}$

Crack Spacing Distribution in SiC./CAS: Figure 13 shows representative surface cracking at a stress level of $350 \mathrm{MPa}$. Figure 14 shows the standard deviation of crack spacing as a

"The compliance change which accompanies initial microcracking is typically not sufficient in extent to be detected by use of conventional load and displacement transducers.

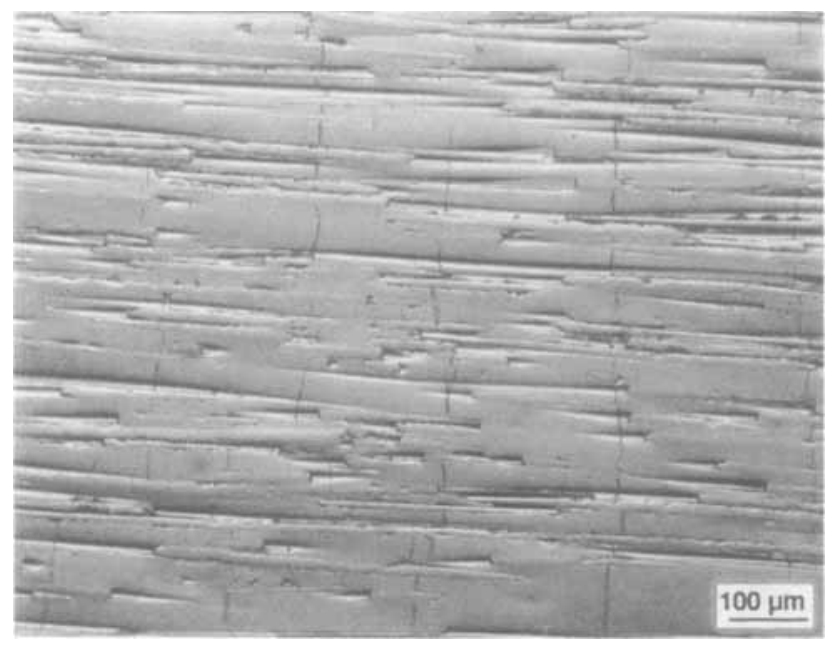

Fig. 13. Surface replica showing microcracking in a $\mathrm{SiC}_{1} / \mathrm{CAS}$ composite after loading to $350 \mathrm{MPa}$. The loading direction is from left to right.

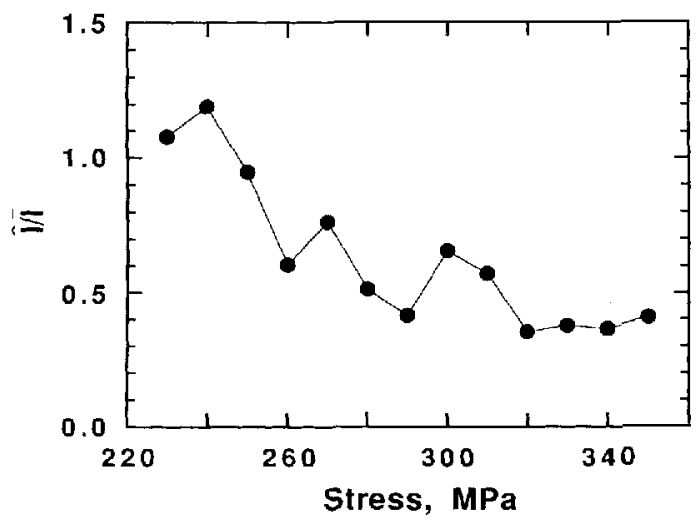

Fig. 14. Standard deviation of the crack spacing.

function of applied stress. Although cracking was first observed at a stress of $160 \mathrm{MPa}$, the number of cracks found for stresses below $230 \mathrm{MPa}$ was not sufficient to obtain accurate statistics for crack spacing. Comparing Figs. 8 and 14 shows that both the experimental and analytically determined standard deviations in mean crack spacing approach the same limiting value of approximately 0.375 . In agreement with the theoretical results obtained for a large standard deviation in crack spacing (see Section III(2)), the experimentally determined crack spacing follows a Weibull plot with an average correlation of 0.97 for applied stresses between 270 and $350 \mathrm{MPa}$.

From the analytical results, the best correlation in matrix crack spacing standard deviation occurred when the standard deviation in matrix strength was approximately 0.4 (Fig. 7). This result indicates the need to include size effects when calculating bounds for the crack spacing. If we know the standard deviation of matrix strength (from a Weibull plot of experimentally determined matrix strength values), we can improve our bounds for crack spacing. Conversely, if we know the statistics for crack spacing, we can estimate the standard deviation of monolithic matrix strength.

\section{Conclusions}

The theoretical analysis shows that matrix strength statistics have a significant effect on the distribution of matrix cracks in a uniaxial composite loaded in tension and that a large variance is to be expected in the crack spacing distribution even when the matrix strength is relatively homogeneous. In the limiting case of a completely homogencous material, the crack spacing distribution tends to an inverse square distribution between $l_{\max }$ and $l_{\max } / 2$.

Experimental measurements of crack spacing distributions for a $[0]_{16} \mathrm{SiC}_{f} / \mathrm{CAS}$ composite show good agreement with the theoretical predictions and suggest that the standard deviation of the matrix strength is of the order of $40 \%$ of the mean strength. With this magnitude of strength variation, the material exhibits a significant size effect and it is essential to take this into account in estimating the mean crack spacing from the corresponding mean matrix propertics.

\section{APPENDIX A}

\section{Stresses and Debonding Length in the Model}

To obtain the shear stress distribution in the presence of matrix cracking an isolated shear-lag model similar to that adopted by Aveston and Kelly ${ }^{4}$ is used. Each fibcr is assumed to be embedded in a matrix cylinder of outer diameter $D_{\mathrm{c}}$ as in Ref. 12. Defining in terms of the actual fiber diameter $d_{l}$ and fiber volume fraction $v_{f}$ gives the correct volume fraction of fibers (see Fig. 2):

$$
D_{\mathrm{c}}=\frac{d_{\mathrm{f}}}{\sqrt{v_{\mathrm{L}}}}
$$


In the analysis it is assumed that the fiber carries only axial load and the matrix transmits constant transverse shear stress. ${ }^{4}$ For force equilibrium of a fiber element in the axial direction

$$
\tau+\frac{d_{\mathrm{f}}}{4} \frac{\mathrm{d} \sigma_{\mathrm{f}}}{\mathrm{d} z^{\prime}}=0
$$

For force equilibrium of the composite in the axial direction

$$
A_{\mathrm{c}} \sigma=A_{\mathrm{f}} \sigma_{\mathrm{f}}+A_{\mathrm{m}} \sigma_{\mathrm{m}}=F
$$

where $\sigma_{\mathrm{m}}$ is the mean stress of the matrix. Differentiating Eq. (A-2) with respect to $z^{\prime}$ gives

$$
\frac{\mathrm{d} \sigma_{\mathrm{m}}}{\mathrm{d} z^{\prime}}=-\frac{v_{\mathrm{f}}}{v_{\mathrm{m}}} \frac{\mathrm{d} \sigma_{\mathrm{f}}}{\mathrm{d} z^{\prime}}
$$

The displacements in the fiber, $u_{\mathrm{f}}$, and on the surface of a representative cylinder, $u_{\mathrm{m}}$, are

$$
u_{\mathrm{m}}-u_{\mathrm{f}}=\frac{\left(D_{\mathrm{c}}-d_{\mathrm{f}}\right) \gamma}{2}=\frac{d_{\mathrm{f}}\left(1 / \sqrt{v_{\mathrm{f}}}-1\right) \tau}{2 G_{\mathrm{m}}}
$$

Differentiating Eq. (A-4) twice and using Hooke's law yields

$$
\frac{1}{E_{\mathrm{m}}} \frac{\mathrm{d} \sigma_{\mathrm{m}}}{\mathrm{d} z^{\prime}}-\frac{1}{E_{\mathrm{f}}} \frac{\mathrm{d} \sigma_{\mathrm{f}}}{\mathrm{d} z^{\prime}}=\frac{d_{\mathrm{f}}\left(1 / \sqrt{\nu_{\mathrm{f}}}-1\right)}{2 G_{\mathrm{m}}} \frac{\mathrm{d}^{2} \tau}{\mathrm{d} z^{\prime 2}}
$$

Substituting Eqs. (A-1) and (A-3) into Eq. (A-5) and defining

$$
\lambda^{2}=\frac{8 G_{\mathrm{m}}}{C d_{\mathrm{l}}^{2} E_{\mathrm{f}}\left(\sqrt{v_{\mathrm{f}}}-v_{\mathrm{f}}\right)}
$$

yields

$$
\frac{\mathrm{d}^{2} \tau}{\mathrm{d} z^{\prime 2}}-\lambda^{2} \tau=0
$$

The solution to Eq. $(\mathrm{A}-6)$ is of the form

$$
\tau=A e^{-\lambda z^{\prime}}+B e^{\lambda z^{\prime}}
$$

where $A$ and $B$ are constants. Since $\tau=0$, and assuming that $\lambda l \gg 1$, we find from Eq. $(\mathrm{A}-7)$ that $B=0$ at $z^{\prime} \rightarrow \infty$ (where $z^{\prime}$ is the distance from the crack plane). From Eqs. (A-1) and (A-7)

$$
\sigma_{\mathrm{f}}=\frac{4 A}{\lambda d_{\mathrm{f}}} e^{-\lambda z^{\prime}}+A^{\prime}
$$

where $A^{\prime}$ is a constant. The boundary conditions are

$$
\begin{gathered}
\sigma_{\mathrm{f}}(0)=\frac{\sigma}{v_{f}} \\
\sigma_{\mathrm{f}}\left(\frac{l}{2}\right)=\frac{E_{\mathrm{f}} \sigma}{E}
\end{gathered}
$$

Therefore,

$$
\begin{aligned}
A & =\frac{\lambda d_{\mathrm{f}} C \sigma}{4\left(1-e^{-0.5 \lambda l}\right)} \approx \frac{\lambda d_{\mathrm{f}} C \sigma}{4} \\
A^{\prime} & =\frac{\sigma}{v_{\mathrm{f}}}-\frac{C \sigma}{1-e^{-(0.5 \lambda l}} \approx \sigma \frac{E_{\mathrm{f}}}{E} \\
\tau & =\frac{\lambda d_{\mathrm{f}} \sigma C}{4} e^{-\lambda z^{\prime}}=\frac{\lambda d_{\mathrm{f}}\left(\sigma_{\mathrm{f} f 1}-\sigma_{\mathrm{f}(l / 2)}\right)}{4} e^{-\lambda z^{\prime}}
\end{aligned}
$$

When $\tau(0) \geq \tau_{\mathrm{i}}$ (interfacial strength), debonding occurs and the shear stress distribution along the fiber becomes

$$
\begin{array}{rlrl}
\tau & =\tau_{i} & & \left(0 \leq z^{\prime} \leq \delta\right) \\
& =\tau_{i} e^{\lambda\left(z^{\prime}-\delta\right)} & \left(\frac{l}{2} \geq z^{\prime}>\delta\right)
\end{array}
$$

where $\tau_{\mathrm{f}}$ and $\delta$ are the friction and debonding length, respec- tively. From axial force equilibrium in the fiber

$$
\tau_{f} \delta-\frac{\tau_{\mathrm{i}}}{\lambda} e^{-\lambda(0.5 l-\delta)} \approx \tau_{f} \delta=\frac{d_{\mathrm{f}} C \sigma}{4}-\frac{\tau_{i}}{\lambda}
$$

Thus, the debonding length from one crack plane becomes

$$
\frac{\delta}{d_{\mathrm{f}}}=\frac{C}{4} \frac{\sigma}{\tau_{\mathrm{f}}}-\frac{1}{\lambda d_{\mathrm{f}}} \frac{\tau_{\mathrm{i}}}{\tau_{\mathrm{f}}}
$$

\section{APPENDIX B}

\section{Distribution of Crack Spacings for Homogeneous Matrix Strength}

If the matrix is completely homogeneous in strength, cracks will always form at the midpoint of the cell at the condition defined by Eq. (18) or (21). In this section, we shall determine the spacing distribution that would preserve its form with increasing load under these conditions.

For the distribution to preserve its form, the number of cells having a length between $x$ and $x+\delta x$ must be of the form $P(x) \delta x$, where

$$
P(x)=f(\xi) g\left(l_{0}\right) \quad\left(\frac{l_{0}}{2}<x<l_{0}\right)
$$

for all loads, $l_{0}$ is the maximum crack spacing $l_{\max }$ corresponding to the current load and $\xi=x / l_{0}$.

Suppose we now increase the load slightly so that $l_{\max }=$ $l_{0}-\epsilon$. Since the matrix has homogeneous strength, the only effect of this change will be to cause additional cracks at the midpoints of all those cells of length $l_{0}-\epsilon<x<l_{0}$. Thus, for all remaining values of $x$ we must have $\mathrm{d} P / \mathrm{d} l_{0}=0$ leading to the differential cquation

$$
l_{0} g^{\prime}\left(l_{0}\right) f(\xi)-\xi f^{\prime}(\xi) g\left(l_{0}\right)=0
$$

This equation is satisfied for all $x, l_{0}$ if and only if $f(\xi)=A \xi^{n}$ and $g\left(l_{0}\right)=B l_{n}^{n}$, leading to the general form

$$
P(x)=C x^{n} \quad\left(\frac{l_{0}}{2}<x<l_{0}\right)
$$

where $A, B$, and $C$ are arbitrary constants. The total number of cells at some given $l_{0}$ is then

$$
N\left(l_{0}\right)=\int_{l_{\mid / 2} / 2}^{l_{0}} P(x) \mathrm{d} x=\frac{C l_{0}^{n+1}}{n+1}\left(1-\frac{1}{2^{n+1}}\right)
$$

Finally, to determine the exponent $n$ we note that there are $P\left(l_{0}\right) \epsilon$ cells in the range $l_{0}<x<l_{0}-\epsilon\left(\epsilon \ll l_{0}\right)$, each of which will lead to the formation of a new crack and hence a new cell when $l_{0}$ is reduced to $l_{0}-\epsilon$. It follows that $P\left(l_{0}\right)=$ $-N^{\prime}\left(l_{0}\right)$ and hence

$$
C l_{0}^{n}=-C l_{0}^{n}\left(1-\frac{1}{2^{n+1}}\right)
$$

which requires that $n=-2$. Thus, the only distribution which preserves its form under the process is $P(x)=C x^{-2}$, $l_{0} \geq x \geq l_{0} / 2$, and the normalized cumulative distribution function is

$$
\begin{aligned}
& \Phi(x)=\frac{1}{N\left(l_{0}\right)} \int_{l_{0} / 2}^{l_{0}} P(x) \mathrm{d} x=2-\frac{l_{0}}{x} \\
& \left(l_{0} \geq x \geq \frac{l_{0}}{2}\right)
\end{aligned}
$$

We also note that the mean crack spacing is

$$
\bar{l}=\frac{1}{N\left(l_{0}\right)} \int_{l_{(! / 2}}^{l_{0}} x P(x) \mathrm{d} x=l_{0} \ln 2
$$


and the standard deviation is

$$
\hat{l}=\left[\frac{1}{N\left(l_{0}\right)} \int_{l_{0} / 2}^{t_{11}}(x-\bar{l})^{2} P(x) \mathrm{d} x\right]^{1 / 2}=0.2017 \bar{l}
$$

\section{Nomenclature}

$A, A_{\mathrm{m}}, A_{\mathrm{f}}$ Cross-sectional area (composite, matrix, and fiber)

$b \quad$ Weibull modulus of matrix

C Dimensionless constant, $v_{\mathrm{m}} E_{\mathrm{m}} / v_{\mathrm{f}} E$

$d_{\mathrm{f}} \quad$ Fiber diameter

$\delta \quad$ Fiber debond length

$E, E_{\mathrm{m}}, E_{\mathrm{f}} \quad$ Tensile moduli of composite, matrix, and fiber, respectively

\section{$F \quad$ Applied force}

$G_{\mathrm{m}} \quad$ Matrix shear modulus of matrix

$l^{\mathrm{m}} \quad$ Matrix crack spacing

I Mean crack spacing

$\hat{l} \quad$ Standard deviation of crack spacings

$l_{\max } \quad$ Maximum crack spacing

$P(V, S) \quad$ Probability that a volume $V$ does not contain any defects weaker than $S$

$S_{0} \quad$ Reference strength corresponding to a volume, $V_{0}$, such that $P\left(V_{0}, S_{0}\right)=0.368$

$S_{\mathrm{m}} \quad$ Matrix tensile fracture strength

$\bar{S}^{m} \quad$ Mean matrix strength

$\hat{S} \quad$ Standard deviation of matrix strength

$\sigma \quad$ Composite stress

$\sigma_{\mathrm{m}} \quad$ Mean matrix stress

$\sigma_{0} \quad$ Composite stress when matrix cracking occurs

$\sigma_{1} \quad$ Composite stress when interface debonding starts

$\sigma_{2} \quad$ Composite stress when matrix cracking ceases

$\sigma_{i} \quad$ Stress at node $i$

$\sigma_{i}^{*} \quad$ Stress at node $i$ due to a unit load

$\tau$ Interfacial shear stress

$\tau_{1} \quad$ Frictional shear stress

$\tau_{i} \quad$ Interfacial shear strength

$u \quad$ Displacement

$v_{\mathrm{f}}, v_{\mathrm{m}} \quad$ Volume fractions of fiber and matrix, respectively

\section{References}

B.W. Rosen, "Tensile Failure of Fibrous Composites," $A I A A$ J, 2 [11] 1985-91 (1964).

2J. Aveston, G. A. Cooper, and A. Kelly, "Single and Multiple Fracture", pp. 15-26 in The Properties of Fiber Composites, Conferenec on Proceedings, National Physical Laboratory. Guildford IPC Science and Technology Press, Surrey, England, 1971.
G. A. Cooper, and J.M. Silwood, "Multiple Fracture in a Steel Reinforced Fpoxy Resin Composite," J. Mater. Sci., 7, 325-33 (1972).

'J. Aveston and A. Kelly, "Theory of Multiple Fracture of Fibrous Composites," J. Mater. Sci, 8, 352-62 (1973).

J.W. Hutchinson and H.M. Jensen, "Models of Jiber Debonding and Pullout in Brittle Composites with Friction," Mech. Mater., 9, 139-63 (1990).

"H.T. Halin and S.W. Tsai, "On the Behavior of Composite Laminates after Initial railures," J. Compos. Mater., 8, 288-305 (1974).

${ }^{\top}$ T. Mah and K.S. Mendiratta, "Room-Temperature Mechanical Behavior of Fiber-Reinforced Ceramic-Matrix Composites," J. Am. Ceram. Soc., 68 [1] C-27-C-30 (1985)

${ }^{8}$ A. G. Evans and R. M. McMeeking, "On the Toughening of Ceramics by Strong Reinforcements," Acta Metall, 34 [12] 2435-41 (1986).

"V. Gopalaratnam and S. Shah, "Failure Mechanisms and Fracture of Fiber Reinforced Concrete"; pp. 1-25 in Fiber-Reinforced Concrete Properties and Appltcations. Edited by S. P. Shah and G. B. Batson. American Concrete Institute, Detroit, MI. 1987

${ }^{10}$ N. J. Pagano and L. R. Dharani, "Failure Modes in Unidirectional Brittle Matrix Composites (BMC)," Ceram. Eng. Sci. Proc., 8 [7-8] 626-29 (1987).

${ }^{11}$ V.C. Nardone and K. M. Prewo, "Tensile Performance of Carbon-FibreReinforced Glass," J. Mater. Sci, 23, $168-80$ (1988)

${ }^{12}$ D. B. Marshall, B. N. Cox, and A.G. Evans, "The Mechanics of Matrix Crack ing in Brittle-Matrix Fiber Compasites," Acta Metall., 33 [11] 2013. 21 (1985).

${ }^{13}$ B. Budiansky, J.W. Hutchinson, and A. G. Evans, "Matrix Fracture in Fiber-Rcinforced Ceramics," J. Mech. Phys. Solids, 34 [2] 167-89 (1986)

${ }^{14}$ D. B. Marshall and B. N. Cox, "Tensile Fracture of Brittle Matrix Composites: Influence of Fiber Strength," Acta Mctall, 35 [11] 2607-19 (1987).

${ }^{15} \mathrm{M}$. Sutcu, "Statistical ribre Failure and Single Crack Behaviour in Uniaxially Reinforced Ceramic Composites," J. Mater. Sci, 23, 928-33 (1988).

M. D. Thoulcss and A. G. Evans, "Effect of Pull-Out on the Mechanical Propertics of Ceramic-Matrix Composites," Acta Metall., 36 [3] 517-22 (1988).

R. J. Kerans, R.S. Hay, N. J. Pagano, and T. A. Parthasarathy, "The Role of the Fiber-Matrix interface in Ceramic Composites," Am. Ceram. Soc. Bull., 68 [2] 429-42 (1989).

${ }^{*} \mathrm{~K}$ M. Prewo, B Johnson, and S Starrett, "Silicon Carbide FibreReinforced Glass-Ccramic Composite Tensile Behaviour at Elevated Temperature," J. Mater. Sci., 24, 1373-79 (1989).

${ }^{14}$ A. G. Evans, "Perspective on the Development of High-Toughness Ceramics," J. Am. Ceram. Soc., 73 [2] 187-206 (1990).

${ }^{21}$ J.W. I Lolmes, " $\wedge$ Technique for Tensile Fatigue and Creep Testing of Fiber-Reinforced Ceramics," J. Compos. Mater, in press.

${ }^{21}$ S. Shuler and J.W. Holmes, "Influence of Frequency on the Fatigue of a $\mathrm{C}_{\mathrm{F}} / \mathrm{SiC}$ Composite Behavior;" unpublished work.

${ }^{22}$ Annual Book of Standards, Vol. 3.01; p. 757. American Society for Testing and Matcrials, Philadelphia, PA, 1988.

${ }_{2 D}$ D. A. Larsen, Corning Glass Works, Corning, N Y; personal communication, September 1990.

${ }^{24}$ S.W. Wang and A. Parvizi-Majidi, "Mechanical Behavior of Nicalon in Fiber-Reinforced Calcium Aluminosilicatc Matrix Composites," Ceram. Eng. Sci. Proc., 11 [9 10] 1607 $16(1990)$.

${ }^{25}$ I.W. Holmes and S.F. Shuler, "Temperature Rise during Fatigue of Fibre-Reinforced Ceramics," J. Mater. Sci. Lett., 9, 1290-91 (1990).

${ }^{2 n} \mathrm{~J} . \mathrm{W}$. Holmes and C. Cho. "Frictional Heating in a Unidirectional FiberReinforced Ceramic Compositc," J. Mater. Sci. Lett., in press.

${ }^{27} \mathrm{C}$. Cho, J.W. Holmes, and I. R. Barber, "Estimation of Interfacial Shea in Ceramic Composites from Frictional Heating Measurements," J. Am. Ceram. Soc. 74 [11] 2802-808 (1991).

${ }^{2 \mathrm{~J}} \mathrm{~J}$.W. Holmes and C. Cho, "Experimental Observations of Frictional Heating in Fiber-Reinforced Ccramics," J. Am. Ceram. Soc., in press. 